\title{
Custos atribuíveis a obesidade, hipertensão e diabetes no Sistema Único de Saúde, Brasil, 2018
}

\author{
Eduardo Augusto Fernandes Nilson, ${ }^{1}$ Rafaella da Costa Santin Andrade, ${ }^{1}$ Daniela Aquino de Brito ${ }^{1}$ e \\ Michele Lessa de Oliveira²
}

Como citar Nilson EAF, Andrade RCS, Brito DA, Oliveira ML. Custos atribuíveis a obesidade, hipertensão e diabetes no Sistema Único de Saúde, Brasil, 2018. Rev Panam Salud Publica. 2020;44:e32. https://doi.org/10.26633/RPSP.2020.32

RESUMO Objetivo. Estimar os custos atribuíveis a hipertensão arterial, diabetes e obesidade no Sistema Único de
Saúde (SUS) no Brasil em 2018 .
Métodos. Realizou-se uma estimativa dos custos atribuíveis a doenças crônicas não transmissíveis a partir
dos riscos relativos e das prevalências populacionais de hipertensão, diabetes e obesidade, considerando
custos de hospitalizações, procedimentos ambulatoriais e medicamentos distribuídos pelo SUS para tra-
tamento dessas doenças. As informações de custo foram obtidas nos sistemas de informação em saúde
disponíveis no SUS. A análise explorou os custos das doenças segundo sexo e idade na população adulta.
Resultados. Os custos totais de hipertensão, diabetes e obesidade no SUS alcançaram 3,45 bilhões de reais
(R\$) (IC95\%: 3,15 a 3,75) em 2018, ou seja, mais de 890 milhões de dólares (US\$). Desses custos, $59 \%$ foram
referentes ao tratamento da hipertensão, $30 \%$ ao do diabetes e $11 \%$ ao da obesidade. No total, $72 \%$ dos
custos foram com indivíduos de 30 a 69 anos de idade e $56 \%$, com mulheres. Considerando separadamente
a obesidade como fator de risco para hipertensão e diabetes, os custos atribuíveis a essa doença chegaram
a R\$ 1,42 bilhão (IC95\%: 0,98 a 1,87), ou seja, $41 \%$ dos custos totais.
Conclusões. As estimativas dos custos atribuíveis às principais doenças crônicas associadas à alimenta-
ção inadequada evidenciam a grande carga econômica dessas doenças para o SUS. Os dados mostram a
necessidade de priorizar políticas integradas e intersetoriais para a prevenção e o controle da hipertensão,
do diabetes e da obesidade e podem apoiar a defesa de intervenções como medidas fiscais e regulatórias
para alcançar os objetivos da Década de Ação das Nações Unidas sobre Nutrição.

Palavras-chave Doenças não transmissíveis; custos e análises de custo; política pública; obesidade; diabetes mellitus; hipertensão; Brasil.

As doenças crônicas não transmissíveis (DCNTs) são a principal causa de morte e de adoecimento no mundo, tendo as dietas inadequadas entre seus maiores fatores de risco. Além disso, as DCNTs representam uma grande carga para os sistemas de saúde, as sociedades e as economias nacionais devido a seu crescente custo (1-3).

Nesse sentido, a Década de Ação sobre a Nutrição das Nações Unidas (2016-2025) convocou os países membros a se comprometerem com o enfrentamento de todas as formas de má nutrição, com destaque para desnutrição, carências nutricionais específicas, sobrepeso e obesidade e DCNTs associadas

\footnotetext{
1 Ministério da Saúde, Coordenação-Geral de Alimentação e Nutrição, Brasília (DF), Brasil. $₫$ Eduardo Augusto Fernandes Nilson, eduardo@saude.gov.br
}

à alimentação, inclusive hipertensão arterial e diabetes mellitus (4). Por seus efeitos negativos diretos na saúde, somados aos efeitos indiretos resultantes de doenças crônicas associadas, a má nutrição representa uma carga dupla para os sistemas de saúde. $\mathrm{O}$ aumento do consumo de alimentos ultraprocessados em diversas regiões, inclusive na América Latina, e o excesso de sódio, gorduras e açúcar nas dietas representam um desafio às políticas de saúde como causas diretas de doenças crônicas $(5,6)$.

O conhecimento acerca dos custos atribuíveis a doenças pode subsidiar o aprimoramento e a priorização das políticas

\footnotetext{
2 Ministério da Educação, Fundo Nacional de Desenvolvimento da Educação (FNDE), Brasília (DF), Brasil.
} 
de prevenção e enfrentamento das DCNTs, além de fortalecer a defesa de intervenções mais robustas para prevenção dessas doenças, inclusive medidas fiscais e regulatórias. No âmbito da Década, as análises econômicas se somam às evidências epidemiológicas como embasamento para implementar eixos de ação, fortalecendo, por exemplo, as iniciativas nutricionais nos sistemas de saúde, a educação alimentar e nutricional, a construção de ambientes saudáveis que favoreçam escolhas alimentares saudáveis, a ampliação dos investimentos em nutrição e a governança e prestação de contas em nutrição.

Atualmente, o excesso de peso é observado em mais da metade dos brasileiros adultos; a obesidade afeta 16,8\% dos homens e $24,4 \%$ das mulheres (7). A prevalência de obesidade, hipertensão arterial e diabetes cresce gradualmente na população adulta do país (6), variando de 6,6 a 9,4\% para diabetes nos adultos (8) e chegando a 32,3\% para hipertensão arterial (9).

Em paralelo, as DCNTs associadas à alimentação inadequada têm grande impacto direto e indireto sobre a mortalidade precoce no país. Entre as 10 principais causas de morte prematura (de 30 a 69 anos), tanto para mulheres quanto para homens, estão as doenças cardiovasculares, o diabetes, as doenças renais crônicas e alguns cânceres associados à dieta inadequada (10). Em relação a mortes relacionadas a doenças evitáveis no Brasil, as DCNTs estão entre as causas mais prevalentes, com destaque para as doenças cardiovasculares e o diabetes, que totalizaram 72,9 mortes por 100 mil habitantes em 2013 (11).

Embora as evidências acerca do impacto econômico das doenças crônicas nos países de baixa e média renda precisem ser expandidas, cresceu o número de estudos que tratam da contribuição das doenças cardiovasculares e da obesidade (1216). Os estudos sobre o custo da obesidade complementam as informações epidemiológicas tradicionais ao contabilizar o sacrifício econômico devido ao tratamento da doença, aumentando a sensibilidade dos gestores e do público em geral para a adoção de medidas preventivas. Os estudos também permitem estabelecer prioridades de investigação, monitoramento e avaliação, revelando, por exemplo, áreas ou grupos populacionais de maior despesa que requerem intervenção, além de apoiar o processo de busca de eficiência para os sistemas de financiamento da saúde (17).

No Brasil, os custos estimados de doenças cardiovasculares aumentaram em $17 \%$ de 2010 a 2015, alcançando $\mathrm{R} \$ 37,1$ bilhões no ano de 2015 (US\$ 9.6 bilhões), incluindo os custos estimados por morte prematura, os custos diretos com internações e as perdas de produtividade relacionados à doença (18). De 2010 a 2016, os gastos do Sistema Único de Saúde (SUS) atribuíveis ao diabetes com doenças renais crônicas quase dobraram, passando de US\$1,4 milhão para US\$2,6 milhões. Em 2011, os gastos hospitalares e ambulatoriais do SUS com obesidade chegaram a US\$269,6 milhões, dos quais quase $24 \%$ eram atribuíveis à obesidade mórbida $(14,15)$.

Grande parte dos gastos com tratamento de doenças no SUS, um sistema de saúde universal e gratuito, se concentra nos atendimentos ambulatoriais e hospitalares, assim como na dispensação de medicamentos para controle de doenças crônicas pelo Programa Farmácia Popular (19). Conhecer os custos das doenças permite dimensionar a magnitude de sua carga para o sistema de saúde e para a economia, assim como avaliar o impacto de políticas implementadas e subsidiar a tomada de decisões ao permitir a comparação da economicidade de distintas medidas.
Diante desse cenário, o objetivo deste estudo foi estimar os custos atribuíveis a hipertensão arterial, diabetes mellitus e obesidade no contexto do SUS no Brasil, considerando internações hospitalares, atendimentos ambulatoriais e gastos com medicamentos em 2018.

\section{MATERIAIS E MÉTODOS}

\section{Custo da doença}

Este estudo de avaliação econômica foi conduzido utilizando uma metodologia de estimação top-down dos custos diretos de doenças adaptada ao Brasil, na perspectiva do SUS. A metodologia se baseia na estimativa da fração atribuível por sexo e grupo etário da hipertensão arterial, do diabetes e da obesidade em função de desfechos específicos. A estimativa da fração atribuível de cada doença foi calculada a partir da prevalência dessa doença na população brasileira adulta e dos riscos relativos estabelecidos para essas doenças primárias e seus desfechos na literatura científica $(20,21)$. O estudo amplia e atualiza estimativas do custo atribuível às principais DCNTs associadas à alimentação, como obesidade e diabetes, e apresenta estimativas inéditas dos custos atribuíveis à hipertensão arterial no SUS $(14,15)$.

\section{Fração atribuível da doença}

Calculou-se o risco atribuível populacional (RAP) para cada doença primária (obesidade, diabetes ou hipertensão arterial) e para seus respectivos desfechos a partir das prevalências e do risco relativo para cada desfecho. O RAP estima a proporção de casos do desfecho associada ao fator de risco ou à doença primária: $\mathrm{RAPi}=\mathrm{P}(\mathrm{RR} i-1) /[\mathrm{P}(\mathrm{RR} \mathrm{i}-1)+1]$, onde $\mathrm{P}=$ prevalência da doença (obesidade, hipertensão arterial ou diabetes) e RRi = risco relativo para desfecho associado à doença avaliada.

\section{Riscos relativos}

Os riscos relativos utilizados no estudo foram obtidos na literatura. Foram identificados e selecionados estudos observacionais, revisões sistemáticas e metanálises que apresentassem dados sobre custos e carga da doença no Brasil e no mundo, com foco na associação entre diabetes, doenças cardiovasculares e doença renal crônica; hipertensão arterial e doenças cardiovasculares; e obesidade, hipertensão arterial, diabetes, cânceres, doenças cardiovasculares (controlando e não controlando a hipertensão arterial), asma, doenças do aparelho digestivo e doenças osteomusculares.

Foram examinadas publicações internacionais e nacionais nas bases Medline, Evidence-Based Medicine Reviews, Cochrane Database of Systematic Reviews, Lilacs, SciELO e Pubmed e considerados os artigos publicados de 1990 a 2019. Os estudos foram sumarizados, sendo as doenças identificadas pelos códigos da Décima Revisão da Classificação Internacional de Doenças (CID-10), como mostra a tabela 1 (22-31).

\section{Dados de custo do SUS}

Os dados de custos são originários dos registros administrativos consolidados anualmente nos sistemas de informação em saúde do SUS (Sistema de Informações Ambulatoriais, SIA; 
TABELA 1. Sumário das principais variáveis utilizadas para estimar os custos atribuíveis das doenças crônicas não transmissíveis

\begin{tabular}{|c|c|c|}
\hline Variáveis & Código da CID-10 & Fonte ${ }^{\mathrm{a}}$ \\
\hline Custos diretos & - & \\
\hline Hospitalizações & - & SIH-SUS 2018 \\
\hline Procedimentos ambulatoriais & - & SIA-SUS 2018 \\
\hline Medicamentos & - & \\
\hline Prevalências & - & \\
\hline Hipertensão arterial & - & PNS 2013 \\
\hline Diabetes mellitus & - & PNS 2013 \\
\hline Obesidade & - & PNS 2013 \\
\hline \multicolumn{3}{|l|}{ Hipertensão arterial } \\
\hline Doença isquêmica do coração & $120-125$ & (24) \\
\hline Acidente vascular cerebral & $160-169$ & (24) \\
\hline Doenças hipertensivas & $|10-| 15$ & (24) \\
\hline Insuficiência cardíaca & 150 & (24) \\
\hline Embolia pulmonar & 126 & (24) \\
\hline Doença reumática do coração & $105-109$ & (24) \\
\hline Aneurisma de aorta & 171 & (24) \\
\hline \multicolumn{3}{|l|}{ Diabetes mellitus } \\
\hline Doença renal crônica & & (23) \\
\hline Doenças cardiovasculares & $\begin{array}{c}\text { I05-I15, I20-I26, I50, } \\
\text { I60-I69, I71 }\end{array}$ & (22) \\
\hline \multicolumn{3}{|l|}{ Obesidade } \\
\hline Diabetes mellitus & E11 & (25) \\
\hline Hipertensão arterial & $|10-| 15$ & $(31)^{b},(25)^{c}$ \\
\hline Acidente vascular cerebral & $160-169$ & $(31)^{b},(25)^{c}$ \\
\hline Embolia pulmonar & 126 & $(31)^{b},(25)^{c}$ \\
\hline $\begin{array}{l}\text { Insuficiência cardíaca } \\
\text { congestiva }\end{array}$ & 150 & $(25)$ \\
\hline Doença isquêmica do coração & $120-125$ & $(31)^{\mathrm{B}},(25)^{\mathrm{C}}$ \\
\hline Câncer esofágico & C15 e D03 & $(25)$ \\
\hline Câncer de pâncreas & D13.6 e D13.7 & (25) \\
\hline Câncer de pele (melanoma) & $\mathrm{C} 43$ e D03 & (26) \\
\hline Câncer de mama & C50, D05, D24 e D48.6 & (25) \\
\hline Câncer de endométrio & $\begin{array}{c}\text { C54.1, C55, D07.0 e } \\
\text { D39.0 }\end{array}$ & (25) \\
\hline Câncer de ovário & C56, D27 e D39.1 & (25) \\
\hline Câncer de vesícula biliar & C23, C24 E D13.5 & (27) \\
\hline Câncer de tiroide & C73 e D44.0 & (26) \\
\hline Câncer renal & C64-66, D30.0-D30.2 & (25) \\
\hline Câncer de cólon & C18 e D12.0-D12.6 & (30) \\
\hline Câncer de reto & C19, C20 e D12.7-D12.9 & (26) \\
\hline Câncer gástrico & C16, D00.2 e D13.1 & (28) \\
\hline Linfoma não-Hodgkin & C82, C83 e C85 & (26) \\
\hline Mieloma múltiplo & $\mathrm{C} 90$ & (26) \\
\hline Leucemia & C91-C95 & (26) \\
\hline Colelitíases e colecistites & K80 e K81 & (26) \\
\hline Osteoartrites & M15-M19 & (25) \\
\hline Dorsalgia & M54 & (25) \\
\hline Asma & $\mathrm{J} 45$ & (25) \\
\hline Pancreatite & K85 e K86 & (29) \\
\hline
\end{tabular}

e Sistema de Informações Hospitalares, SIH) para as doenças avaliadas, identificados por CID-10 e desagregados por sexo e idade. Os custos de tratamento das doenças primárias (hipertensão, diabetes e obesidade) foram considerados integralmente e em valores nominais para o ano de 2018, primeiramente estimando os custos totais atribuíveis às três doenças em conjunto e, em seguida, estimando separadamente os custos atribuíveis à obesidade, incluindo seu impacto como fator de risco para diabetes e hipertensão e para outros desfechos relacionados à obesidade.

Além disso, foram obtidos dados administrativos consolidados de 2017 do Programa Farmácia Popular, assumindo a manutenção dos valores para 2018, para avaliar os custos por medicamento para hipertensão arterial, diabetes e asma, desagregados por idade e sexo. Os valores em dólares (US\$) foram convertidos de acordo com a cotação de 31 de dezembro de 2018 (US\$ $1=\mathrm{R} \$ 3,875)$.

\section{Prevalências dos fatores de risco}

As prevalências de hipertensão, diabetes e obesidade foram obtidas da Pesquisa Nacional de Saúde (PNS) de 2013, o mais recente inquérito populacional brasileiro representativo com adultos (18 anos ou mais de idade) que inclui medidas antropométricas, aferição da pressão arterial e coleta de material biológico (sangue e urina) para análises laboratoriais $(7,8,32)$.

\section{RESULTADOS}

Em 2018, houve 1829779 internações por causas associadas à hipertensão arterial, ao diabetes e à obesidade no SUS. Isso corresponde a aproximadamente $16 \%$ do total de internações hospitalares no SUS no período, resultando em um custo total de R \$ 3,84 bilhões. Os custos ambulatoriais totais com as mesmas doenças no SUS somaram R\$ 166 milhões no ano de 2018, e os gastos do Programa Farmácia Popular com medicamentos para hipertensão, diabetes e asma totalizaram R 2,31 bilhões. Os custos diretos atribuíveis a hipertensão arterial, diabetes e obesidade no Brasil totalizaram R \$ 3,45 bilhões, ou seja, US\$ 890 milhões, considerando gastos do SUS com hospitalizações, procedimentos ambulatoriais e medicamentos (tabela 2).

Comparando os custos por tipo de gasto no SUS, a maioria se deveu ao fornecimento de medicamentos a pessoas com obesidade, diabetes e hipertensão arterial (58,8\%), seguido por hospitalizações $(34,6 \%)$ e atendimentos/procedimentos ambulatoriais $(6,6 \%)$. Considerando sexo e idade, $56 \%$ dos custos totais foram com o sexo feminino e mais de $70 \%$ dos custos foram com pessoas de 20 a 69 anos de idade.

A hipertensão arterial foi responsável por $59 \%$ do custo direto (mais de R\$ 2 bilhões por ano), enquanto o diabetes correspondeu a $30 \%$ e a obesidade, a $11 \%$ (desconsiderando os custos de hipertensão e diabetes atribuíveis à obesidade), com pouca variação de acordo com o sexo. Considerando somente as internações hospitalares, os custos atribuíveis a essas três doenças somaram $9,8 \%$ de todos os gastos com hospitalizações de adultos no país. Ainda é importante considerar os impactos da obesidade como fator de risco para hipertensão e diabetes em adultos, os quais não foram considerados nos custos atribuíveis à obesidade na análise anterior para evitar duplicidade de contagem.

No Brasil, excesso de peso e obesidade são fatores de risco importantes para hipertensão e diabetes, coexistindo, assim, em 
TABELA 2. Custos atribuíveis a hipertensão arterial, diabetes mellitus e obesidade para hospitalizações, procedimentos ambulatoriais e medicamentos em adultos no SUS ${ }^{a}$, Brasil, 2018

\begin{tabular}{lrrr} 
& \multicolumn{3}{c}{ Custos atribuiveis (R\$) } \\
\cline { 2 - 4 } Homens & \multicolumn{1}{c}{ Média } \\
Obesidade & 143407601,37 & 50919058,32 & 246041460,35 \\
Hipertensão & 910559025,82 & 895807761,24 & 925559380,27 \\
Diabetes & 465179453,92 & 451228821,06 & 481018494,20 \\
$\quad$ Total & 1519146081,11 & 1397955640,63 & 1652619334,82 \\
Mulheres & & & \\
Obesidade & 228281613,65 & 87504236,98 & 374909249,25 \\
Hipertensão & 1118864583,54 & 1109470438,20 & 1128465349,78 \\
Diabetes & 583068051,57 & 561789354,19 & 598706091,01 \\
$\quad$ Total & 1930214248,76 & 1758764029,37 & 2102080690,04 \\
Total & & & \\
Obesidade & 371689215,03 & 138423295,30 & 620950709,60 \\
Hipertensão & 2029423609,36 & 2005278199,44 & 2054024730,05 \\
Diabetes & 1048247505,49 & 1013018175,26 & 1079724585,21 \\
$\quad$ Total & 3449360329,88 & 3156719670,00 & 3754700024,86 \\
\hline Excluindo os desfechos mediados por hipertensão e diabetes nos custos de obesidade.
\end{tabular}

grande parte dos diabéticos e hipertensos. Segundo dados da PNS 2013, mais de um terço dos diabéticos (37,0\%; IC95\%: 34,2 a $39,8 \%$ ) e dos hipertensos (36,3\%; IC95\%: 34,8 a $37,7 \%$ ) eram obesos. Além disso, 75,2\% dos diabéticos (IC95\%: 72,8 a 77,5\%) e 74,4\% dos hipertensos (IC95\%: 73,0 a 75,7\%) apresentavam excesso de peso no Brasil.

Assim, agregar a obesidade a essas comorbidades permite uma estimativa mais completa do impacto econômico da obesidade no SUS. Com a incorporação dos custos da obesidade como fator de risco para hipertensão e diabetes, os custos totais atribuíveis à obesidade aumentam para R 669 milhões em hospitalizações e gastos ambulatoriais; e para R\$ 722 milhões em gastos com medicamentos, ou seja, um total de $\mathrm{R} \$ 1,39$ bilhão em 2018 (tabela 3). Mais de $60 \%$ dos gastos totais atribuíveis à obesidade foram com mulheres, tendo em vista a maior prevalência de obesidade e o maior risco relativo de alguns desfechos, particularmente doenças cardiovasculares, no sexo feminino.

\section{DISCUSSÃO}

A associação das DCNTs com alimentação inadequada, assim como com outros fatores de risco - como tabagismo, sedentarismo, consumo de álcool e antecedentes familiares - tem grande e crescente impacto negativo na saúde das populações, reforçando a causalidade múltipla das DCNTs. As doenças cardiovasculares, o diabetes, as doenças renais crônicas e alguns cânceres associados a riscos dietéticos estão entre as principais causas de mortes prematuras e evitáveis no Brasil. Além disso, sobrecarregam os sistemas de saúde em função dos gastos com tratamentos e oneram a economia com perdas de produtividade. Entretanto, vale notar que as análises apresentadas neste artigo não contabilizam os custos da saúde suplementar no país, nem tampouco os custos econômicos e sociais associados a adoecimento e morte por essas causas.

TABELA 3. Custos totais de hospitalizações, procedimentos ambulatoriais e medicamentos atribuíveis à obesidade, incluindo os custos da obesidade como fator de risco para hipertensão e diabetes, SUS ${ }^{a}$, Brasil, 2018

\begin{tabular}{cr} 
& Custos atribuíveis (R\$) \\
\hline Média & IC 95\%
\end{tabular}

\section{Masculino}

\section{Diabetes}

Hipertensão arterial

Doenças

cardiovasculares

Cânceres

Obesidade

Outras doenças

Total

Feminino

Diabetes

Hipertensão arterial

Doenças

cardiovasculares

Cânceres

Obesidade

Outras doenças

Total

Total

Diabetes

Hipertensão arterial

Doenças

cardiovasculares

Cânceres

Obesidade

Outras doenças

Total

$$
\text { a Incl }
$$
As dietas inadequadas, com baixo consumo de cereais, frutas,
hortaliças e excesso de nutrientes críticos, estão entre os principais fatores de risco para DCNTs na América Latina, incluindo o Brasil. Esses fatores dietéticos estão diretamente associados ao crescimento do consumo de ultraprocessados: de 2000 a 2013, o volume comercializado de ultraprocessados cresceu $43,7 \%$ em todo o mundo e $48 \%$ na América Latina (33), junto com o consumo de sal, açúcar, óleos e gorduras. Para o enfrentamento dessa situação, são exigidas múltiplas políticas.

Nesse sentido, devido às prevalências crescentes das DCNTs, incluindo obesidade, hipertensão e diabetes, os custos aos sistemas de saúde e às economias nacionais aumentam por meio da mortalidade precoce, das aposentadorias precoces, do absenteísmo e do presenteísmo. Com isso, a necessidade de priorizar medidas preventivas aumenta, desde as de caráter individual, familiar e comunitário até as que influenciam os ambientes, em particular os sistemas alimentares. Entre as medidas mais custo-efetivas para o controle dessas DCNTs, estão a redução da porção de alimentos, a regulação da publicidade de alimentos para crianças, a regulação da oferta de alimentos e bebidas não saudáveis nas escolas, o aprimoramento da rotulagem nutricional de alimentos, a taxação de bebidas e alimentos não saudáveis e a reformulação de alimentos processados e ultraprocessados (34). 
No Brasil, grandes avanços foram alcançados na promoção da alimentação saudável, principalmente a partir da revisão do Guia Alimentar para a População Brasileira (35). Contudo, diante da necessidade de deter o crescimento das DCNTs associadas a fatores dietéticos, o avanço de outras medidas que facilitem escolhas alimentares saudáveis em relação às não saudáveis é fundamental.

Nas Américas, vários países se tornaram importantes lideranças regionais e globais na regulação de alimentos. São exemplos o Chile (36), que adotou a rotulagem frontal de alimentos, seguido pelo Peru e Uruguai, e o México, com a taxação de bebidas adoçadas (37). Em relação à redução do sódio, a reformulação de alimentos industrializados avançou por medidas regulatórias na Argentina e voluntariamente no Brasil $(38,39)$.

Ferramentas como as metodologias de custo da doença aplicadas neste estudo podem ser utilizadas em distintos cenários, com informações nacionais oriundas de estudos e inquéritos populacionais. Na ausência de informações nacionais, estimativas internacionais podem ser utilizadas, como o estudo Global Burden of Disease (GBD). Somados às evidências internacionais existentes, dados e estimativas nacionais de mortes e custos atribuíveis podem apoiar de maneira mais decisiva os tomadores de decisão e formuladores de políticas.
Por fim, a construção de políticas mais efetivas depende da ampliação e do fortalecimento de evidências para subsidiar os processos decisórios e fazer prevalecer os interesses de saúde pública, inclusive levando em consideração os aspectos econômicos do processo saúde-doença e seus impactos. No âmbito dos compromissos globais de nutrição, particularmente da Década de Ação sobre a Nutrição, evidências econômicas podem se somar às epidemiológicas existentes e fortalecer a implementação de políticas mais custo-efetivas para o enfrentamento da dupla carga da má nutrição.

Contribuição dos autores. EAFN concebeu o estudo, extraiu os dados, revisou a literatura, analisou os dados e escreveu a versão preliminar do artigo. RCSA e DAB extraíram e analisaram os dados. MLO supervisionou as análises. Todos os autores redigiram o artigo e aprovaram a versão final.

Conflitos de interesse. Nada declarado pelos autores.

Declaração. As opiniões expressas no manuscrito são de responsabilidade exclusiva dos autores e não refletem necessariamente a opinião ou política da RPSP/PAJPH ou da Organização Pan-Americana da Saúde (OPAS).

\section{REFERÊNCIAS}

1. Afshin A, Sur PJ, Fay KA, Cornaby L, Ferrara G, Salama JS, et al. Health effects of dietary risks in 195 countries, 1990-2017: a systematic analysis for the Global Burden of Disease Study 2017. Lancet. 2019;393(10184):1958-72. https://doi.org/10.1016/S01406736(19)30041-8

2. Rasmussen B, Sweeny K, Sheehan P. Economic costs of absenteeism, presenteeism and early retirement due to ill health : a Focus on Brazil. Melbourne: Victoria Institute of Strategic Economic Studies; 2015. Disponível em: https://www.brazilcouncil.org/wp-content/ uploads/2016/04/Econ.-Cost-of-Absenteeism-Presenteeism-andEarly-Retirement-due-to-ill-health-Brazil.pdf Acessado em 28 de janeiro de 2020.

3. Bertram MY, Sweeny K, Lauer JA, Chisholm D, Sheehan P, Rasmussen $\mathrm{B}$, et al. Investing in non-communicable diseases: an estimation of the return on investment for prevention and treatment services. Lancet. 2018;391(10134):2071-8. https://doi.org/10.1016/S01406736(18)30665-2

4. UN General Assembly. United Nations Decade of Action on Nutrition (2016-2025). Work Programme. 2018. https://www.un.org/ nutrition/sites/www.un.org.nutrition/files/general/pdf/mv131 rev1_undoa_wp_rev1_en.pdf. Acessado em 28 de fevereiro de 2020.

5. Portes LH, Machado CV, Turci SRB, Figueiredo VC, Cavalcante TM, Silva VLDCE. Tobacco control policies in Brazil: A 30-year assessment. Cienc Saude Colet. 2018;23(6):1837-48. https://doi. org /10.1590/1413-81232018236.05202018

6. Brasil, Ministério da Saúde. Vigitel Brasil 2018 - Vigilância de Fatores de Risco e Proteção para Doenças Crônicas por Inquérito Telefônico. Brasília: Departamento de Análise em Saúde e Vigilância de Doenças Não Transmissíveis; 2019. Disponível em: https:// portalarquivos2.saude.gov.br/images/pdf/2019/julho/25/vigitelbrasil-2018.pdf Acessado em 28 de janeiro de 2020.

7. Ferreira APS, Szwarcwald CL, Damacena GN. Prevalência e fatores associados da obesidade na população brasileira: estudo com dados aferidos da Pesquisa Nacional de Saúde, 2013. Rev Bras Epidemiol. 2019;22:e190024. http:/ / dx.doi.org/10.1590/1980-549720190024

8. Malta DC, Duncan BB, Schmidt MI, Machado IE, Silva AG, Bernal RTI, et al. Prevalência de diabetes mellitus determinada pela hemoglobina glicada na população adulta brasileira, Pesquisa Nacional de Saúde. Rev Bras Epidemiol. 2019;22(supl 2):E190006.SUPL.2. http:/ /dx.doi.org/10.1590/1980-549720190006.supl.2
9. Malta DC, Santos NB, Perillo RD, Szwarcwald CL. Prevalence of high blood pressure measured in the Brazilian population, National Health Survey, 2013. Sao Paulo Med J. 2016;134(2):163-70. http:// dx.doi.org/10.1590/1516-3180.2015.02090911

10. Brasil, Ministério da Saúde. Saúde Brasil 2018 - Uma análise da situação de saúde e das doenças e agravos crônicos: desafios e perspectivas. Brasília: Secretaria de Vigilância em Saúde; 2019. Disponível em: http://bvsms.saude.gov.br/bvs/publicacoes/ saude_brasil_2018_analise_situacao_saude_doencas_agravos_cro nicos_desafios_perspectivas.pdf Acessado em 28 de janeiro de 2020.

11. Malta DC, Saltarelli RMF, Prado RR, Monteiro RA, Almeida MF. Mortes evitáveis no Sistema Único de Saúde na população brasileira, entre 5 e 69 anos, 2000-2013. Rev Bras Epidemiol. 2018;21(0):e180008. http:/ /dx.doi.org/10.1590/1980-549720180008

12. Gheorghe A, Griffiths U, Murphy A, Legido-Quigley H, Lamptey P, Perel P. The economic burden of cardiovascular disease and hypertension in low- and middle-income countries: A systematic review. BMC Public Health. 2018;18(1):975. https://doi.org/10.1186/ s12889-018-5806-x

13. Rtveladze K, Marsh T, Barquera S, Sanchez Romero LM, Levy D, Melendez G, et al. Obesity prevalence in Mexico: impact on health and economic burden. Public Health Nutr. 2014;17(1):233-9. https://doi.org/10.1017/S1368980013000086

14. Oliveira ML, Santos LMP, Silvada EN. Direct healthcare cost of obesity in brazil: An application of the cost-of-illness method from the perspective of the public health system in 2011. PLoS One. 2015;10(4):e0121160. https:/ / doi.org/10.1371/journal.pone.0121160

15. Goncalves GMR, Silva END. Cost of chronic kidney disease attributable to diabetes from the perspective of the Brazilian Unified Health System. PLoS One. 2018;13(10):e0203992. https://doi.org/10.1371/ journal.pone.0203992

16. Stevens B, Pezzullo L, Verdian L, Tomlinson J, George A, Bacal F. The economic burden of heart conditions in Brazil. Arq Bras Cardiol. 2018;111(1):29-36. https:/ / doi.org/10.5935/abc.20180104

17. Pereira J, Mateus C, Amaral MJ. Custos da obesidade em Portugal. Lisboa: Associação Portuguesa de Economia da Saúde; 1999. Disponível em: https://www.ensinobasico.com/attachments/arti cle/963/obesidade99.pdf Acessado em 28 de janeiro de 2020.

18. Siqueira ASE, Siqueira-Filho AG, Land MGP. Analysis of the Economic Impact of Cardiovascular Diseases in the Last Five 
Years in Brazil. Arq Bras Cardiol. 2017;109(1):39-46. https://doi. org/10.5935/abc.20170068

19. Silva RM, Caetano R. "Farmácia Popular do Brasil" Program: Characterization and evolution between 2004 and 2012. Cienc Saude Colet. 2015;20(10):2943-56. http://dx.doi.org/10. 1590/1413-812320152010.17352014

20. Oliveira ML, Santos LMP, Silva EN. Bases metodológicas para estudos de custos da doença no Brasil. Rev Nutr. 2014;27(5):585-95. http:/ /dx.doi.org/10.1590/1415-52732014000500007

21. Scarborough P, Harrington RA, Mizdrak A, Zhou LM, Doherty A. The Preventable Risk Integrated ModEl and Its Use to Estimate the Health Impact of Public Health Policy Scenarios. Scientifica (Cairo). 2014;2014: 748750. https://doi.org/10.1155/2014/748750

22. Kahleova H, Salas-Salvadó J, Rahelić D, Kendall CW, Rembert E, Sievenpiper JL. Dietary Patterns and Cardiometabolic Outcomes in Diabetes: A Summary of Systematic Reviews. Nutrients. 2019;11(9):E2209. https:/ / doi.org/10.3390/nu11092209

23. Yu MK, Katon W, Young BA. Associations between sex and incident chronic kidney disease in a prospective diabetic cohort. Nephrology (Carlton). 2015;20(7):451-8. https:/ /doi.org/10.1111/nep.12468

24. Lewington S, Clarke R, Qizilbash N, Peto R, Collins R. Age-specific relevance of usual blood pressure to vascular mortality: A metaanalysis of individual data for one million adults in 61 prospective studies. Lancet. 2002;360(9349):1903-13. https://doi.org/10.1016/ s0140-6736(02)11911-8

25. Guh DP, Zhang W, Bansback N, Amarsi Z, Birmingham CL, Anis $\mathrm{AH}$. The incidence of co-morbidities related to obesity and overweight: A systematic review and meta-analysis. BMC Public Health. 2009;9:88. https://doi.org/10.1186/1471-2458-9-88

26. Renehan AG, Tyson M, Egger M, Heller RF, Zwahlen M. Body-mass index and incidence of cancer: a systematic review and meta-analysis of prospective observational studies. Lancet. 2008;371(9612):569-78. https://doi.org/10.1016/S0140-6736(08)60269-X

27. Engeland A, Tretli S, Austad G, Bjørge T. Height and body mass index in relation to colorectal and gallbladder cancer in two million Norwegian men and women. Cancer Causes Control. 2005;16(8):98796. https://doi.org/10.1007/s10552-005-3638-3

28. Yang P, Zhou Y, Chen B, Wan HW, Jia GQ, Bai HL, et al. Overweight, obesity and gastric cancer risk: Results from a meta-analysis of cohort studies. Eur J Cancer. 2009;45(16):2867-73. https://doi. org/10.1016/j.ejca.2009.04.019

29. Martínez J, Sánchez-Payá J, Palazón JM, Suazo-Barahona J, Robles-Díaz G, Pérez-Mateo M. Is obesity a risk factor in acute pancreatitis? A meta-analysis. Pancreatology. 2004;4(1):42-8. https:// doi.org/10.1159/000077025

30. Dai Z, Xu YC, Niu L. Obesity and colorectal cancer risk: A metaanalysis of cohort studies. World J Gastroenterol. 2007;13(31):4199206. https://doi.org/10.3748/wjg.v13.i31.4199

31. Bogers RP, Bemelmans WJE, Hoogenveen RT, Boshuizen HC, Woodward M, Knekt P, et al. Association of overweight with increased risk of coronary heart disease partly independent of blood pressure and cholesterol levels: A meta-analysis of 21 cohort studies including more than 300000 persons. Arch Intern Med. 2007;167(16):1720-8. https:/ / doi.org/10.1001/archinte.167.16.1720

32. Malta DC, Gonçalves RPF, Machado IE, Freitas MIF, Azeredo C, Szwarcwald CL. Prevalence of arterial hypertension according to different diagnostic criteria, National Health Survey. Rev Bras Epidemiol. 2018;21(suppl 1):e180021. https://doi.org/10.1590/ 1980-549720180021.supl.1

33. Pan American Health Organization (PAHO). Ultra-processed food and drink products in Latin America: Trends, impact on obesity, policy implications. Washington, D.C.: PAHO; 2015. Disponível em: http://iris.paho.org/xmlui/bitstream/handle/123 456789/7699/9789275118641_eng.pdf Acessado em 28 de janeiro de 2020.

34. World Health Organization (WHO). Tackling NCDs - "Best buys" and other recommended interventions for the prevention and control of noncommunicable diseases. Genebra: WHO; 2017. Disponível em: http:/ /apps.who.int/iris/bitstream/10665/259232/1/ WHO-NMH-NVI-17.9-eng.pdf?ua=1 Acessado em 02 de março de 2020.

35. Brasil, Ministério da Saúde. Guia Alimentar para a População Brasileira. Brasília, DF: Ministério da Saúde; 2014. Disponível em: https://bvsms.saude.gov.br/bvs/publicacoes/guia_alimentar populacao_brasileira_2ed.pdf Acessado em 28 de fevereiro de 2020.

36. Kanter R, Reyes M, Swinburn B, Vandevijvere S, Corvalán C. The food supply in anticipation of the implementation of the Chilean law of food labeling and advertising. Nutrients. 2019;11(1):E52. https://doi.org/10.3390/nu11010052

37. Grogger J. Soda taxes and the prices of sodas and other drinks: Evidence from Mexico. Am J Agric Econ. 2017;99(2):481-98. https:// doi.org/10.1093/ajae/aax024

38. Castronuovo L, Allemandi L, Tiscornia V, Champagne B, Campbell N, Schoj V. Analysis of a voluntary initiative to reduce sodium in processed and ultra-processed food products in Argentina: the views of public and private sector representatives. Cad Saude Publica. 2017;33(6):e00014316. http://dx.doi.org/ 10.1590/0102-311x00014316

39. Nilson EAF, Spaniol AM, Gonçalves VSS, Moura I, Silva SA, L'Abbé $\mathrm{M}$, et al. Sodium reduction in processed foods in Brazil: Analysis of food categories and voluntary targets from 2011 to 2017. Nutrients. 2017;9(7):E742. https://doi.org/10.3390/nu9070742

Manuscrito recebido em 7 de novembro de 2019. Aceito em versão revisada em 21 de janeiro de 2020 . 


\section{Costs attributable to obesity, hypertension, and diabetes in the Unified Health System, Brazil, 2018}

ABSTRACT Objective. To estimate the cost attributable to arterial hypertension, diabetes and obesity in the Unified Health System of Brazil in 2018.

Method. The study estimated the cost attributable to non-communicable chronic diseases based on relative risk and population prevalence of hypertension, diabetes, and obesity, considering the cost of hospitalizations, outpatient procedures, and medications distributed by the SUS to treat these diseases. Cost data were obtained from SUS information systems. The analysis explored the cost of disease according to sex and age in the adult population.

Results. The total cost of hypertension, diabetes, and obesity in the SUS reached $\mathrm{R} \$ 3.45$ billion (95\% Cl: $3.15-$ 3.75 ) in 2018, that is, more than US\$ 890 million. Of this amount, $59 \%$ referred to the treatment of hypertension, $30 \%$ to diabetes, and $11 \%$ to obesity. The age group from 30 to 69 years accounted for $72 \%$ of the total costs, and women accounted for $56 \%$. When obesity was considered separately as a risk factor for hypertension and diabetes, the cost attributable to this diseases reached $\mathrm{R} \$ 1.42$ billion $(95 \% \mathrm{Cl}: 0.98-1.87)$, i.e., $41 \%$ of the total cost.

Conclusions. The estimates of costs attributable to the main chronic diseases associated with inadequate diet revealed a heavy economic burden of these disorders for the SUS. The data show the need to prioritize integrated and intersectoral policies for the prevention and control of hypertension, diabetes, and obesity, and may support the advocacy for interventions such as fiscal and regulatory measures to ensure that the objectives of the United Nations Decade of Action on Nutrition are met.

Keywords Non communicable disease, costs and cost analysis; public policy; obesity; hypertension; diabetes mellitus; Brazil.

\section{Costos atribuibles a la obesidad, la hipertensión y la diabetes en el Sistema Único de Salud de Brasil, 2018}

RESUMEN Objetivo. Estimar los costos atribuibles a la hipertensión arterial, la diabetes y la obesidad en el Sistema Único de Salud (SUS) de Brasil en el 2018.

Métodos. Se estimaron los costos atribuibles a las enfermedades crónicas no transmisibles a partir de los riesgos relativos y de las tasas de prevalencia poblacional de hipertensión, diabetes y obesidad, teniendo en cuenta los costos de hospitalización, los procedimientos ambulatorios y los medicamentos distribuidos por el SUS para el tratamiento de esas enfermedades. Los datos de costos se obtuvieron en los sistemas de información de salud disponibles en el SUS. En el análisis se exploraron los costos de las enfermedades según el sexo y la edad de la población adulta.

Resultados. Los costos totales atribuibles a la hipertensión, la diabetes y la obesidad en el SUS alcanzaron $\mathrm{R} \$$ 3,450 milliones (IC 95\%: de 3,15 a 3,75) en el 2018, o sea, más de US\$ 890 millones. De esos costos, 59\% correspondió al tratamiento de la hipertensión, 30\% al de la diabetes y $11 \%$ al de la obesidad. En total, $72 \%$ de los costos correspondieron a personas de 30 a 69 años y 56\%, a mujeres. Al considerarse por separado la obesidad como factor de riesgo de hipertensión y diabetes, los costos atribuibles a esa enfermedad alcanzaron R\$ 1.420 millones (IC 95\%: de 0,98 a 1,87), o sea, $41 \%$ del total.

Conclusiones. Las estimaciones de los costos atribuibles a las principales enfermedades crónicas relacionadas con la alimentación inadecuada ponen de manifiesto la pesada carga económica de esas enfermedades para el SUS. Los datos muestran la necesidad de priorizar políticas integradas e intersectoriales para la prevención y el control de la hipertensión, la diabetes y la obesidad, y permiten apoyar la defensa de intervenciones como medidas fiscales y regulatorias para alcanzar los objetivos del Decenio de las Naciones Unidas de Acción sobre la Nutrición.

Palabras clave Enfermedades no transmisibles; costos y análisis de costo; política pública; obesidad; hipertensión; diabetes mellitus; Brasil. 